ISSN = 1980-993X - doi:10.4136/1980-993X
www.ambi-agua.net
E-mail: ambi-agua@agro.unitau.br
Tel.: (12) 3625-4212

\title{
Influência de características físico-químicas da água no transporte de metano para a atmosfera na Lagoa Rodrigo de Freitas, RJ
}

(http://dx.doi.org/10.4136/ambi-agua.972)

\author{
Lia Braz'; Willian José Ferreira²; Marcelo Gomes da Silva³; \\ Plínio Carlos Alvalá ${ }^{4}$; Luciano Marani ${ }^{5}$; Getulio Teixeira Batista ${ }^{6}$; \\ Valiya Mannathal Hamza ${ }^{7}$ \\ 1,2,3,4,5 Instituto Nacional de Pesquisas Espaciais - INPE-São José dos Campos, SP, Brasil, \\ e-mails: lia.braz@hotmail.com, will.ferreira@hotmail.com, engmarcgomes@gmail.com, \\ plinio.alvala@inpe.br, lmarani@gmail.com, \\ ${ }^{6}$ Programa de Pós-Graduação, Universidade de Taubaté, SP, Brasil, \\ e-mail: getulio@agro.unitau.br, \\ ${ }^{7}$ Observatório Nacional, Rio de Janeiro, Brasil, \\ e-mail: hamza@on.br
}

\section{RESUMO}

O acúmulo e a decomposição de matéria orgânica em corpos d'água pode incrementar o potencial de emissão de gases de efeito estufa à atmosfera. Neste trabalho, de modo prospectivo e exploratório, foi avaliada a relação entre características físico-químicas da água e o transporte de metano para a atmosfera na Lagoa Rodrigo de Freitas, RJ, em dias típicos de verão fluminense. Em campanhas realizadas em 2011, foram coletadas 200 amostras para análise da emissão superficial do metano e 30 amostras de água para se verificar a dissolução do gás na coluna d'água, medindo simultaneamente os parâmetros físico-químicos ao longo desta coluna. As medidas de fluxo de metano evidenciaram que a Lagoa é fonte emissora do gás para a atmosfera, com fluxo médio de $33 \pm 10 \mathrm{mgCH}_{4} \mathrm{~m}^{-2} \mathrm{~d}^{-1}$. Na avaliação de como alguns parâmetros limnológicos poderiam influenciar as emissões, observou-se que a altura da lâmina d'água e a temperatura foram os fatores de maior influência nesse processo. A análise do perfil de metano na coluna d'água sugere a existência de uma maior concentração do gás na região dos estratos sedimentares do fundo da lagoa. No entanto, esse metano não chega à superfície, possivelmente, devido à diminuição da taxa de difusão vertical do metano em função do aumento da pressão com a profundidade e também devido à presença de bactérias metanotróficas, que consomem o metano ao longo da coluna d'água.

Palavras-chave: Efeito estufa, gases traços, limnologia.

\section{Influence of physicochemical water characteristics in the transport of methane to the atmosphere at Rodrigo de Freitas lagoon, RJ}

\section{ABSTRACT}

The accumulation and decomposition of organic matter in water bodies can increase the potential emission of greenhouse gases to the atmosphere. In this study, in a prospective and exploratory way, we evaluated the relationship between physicochemical characteristics of water on the transport of methane to the atmosphere at Rodrigo de Freitas lagoon, RJ. In 2011, campaigns were carried out in which 200 samples were collected for analysis of superficial methane emission and 30 water samples were collected to verify the dissolution of 
this gas in the water column, measuring simultaneously both the physicochemical water parameters along this column. The methane flow measurements showed that this lagoon is a source methane emission to the atmosphere with an average flux of $33 \pm 10 \mathrm{mgCH}_{4} \mathrm{~m}^{-2} \mathrm{~d}^{-1}$. The evaluation of limnological parameters that could affect the emission flux revealed that the water depth and temperature were the most influential factors in this process. A profile analysis of methane in the water column suggests the existence of a higher concentration of this gas in the region of the sedimentary strata at the bottom of the lagoon. However, the potential emission may not reach the surface, possibly due to the decrease in the rate of vertical diffusion of methane, as a result of the increase of pressure with depth and the presence of methanotrophic bacterias that consume methane throughout the water column.

Keywords: Greenhouse effect, trace gases, limnology.

\section{INTRODUÇÃO}

Em meio à busca por políticas sustentáveis e eficientes, no sentido de conservar os recursos naturais e proporcionar melhorias ao meio ambiente, o acúmulo de matéria orgânica nos corpos d'água surge como enorme desafio à gestão pública moderna.

$\mathrm{O}$ descarte de dejetos e efluentes de sistemas de esgoto com tratamento deficitário em rios e lagos, aliado à contribuição de águas pluviais com carga orgânica oriunda de áreas urbanizadas, aumenta a importância de estudos que avaliam o potencial de incremento de emissão de gases de efeito estufa (GEE) associado a estes processos.

O metano é o hidrocarboneto mais abundante na atmosfera terrestre, com uma concentração global estimada em 1,8 ppmv (partes por milhão por volume) e é responsável por cerca de $20 \%$ do aquecimento global, onde assume importante papel na regulação térmica do planeta e considerável papel no ciclo do carbono (IPCC, 2007). Somente na década passada, a produção de metano $\left(\mathrm{CH}_{4}\right)$ pela decomposição de matéria orgânica contabilizava $80 \%$ da emissão deste gás para a atmosfera (Dalal e Allen, 2008).

A variabilidade das fontes emissoras de metano torna difícil uma avaliação mais precisa do quanto elas contribuem individualmente. Contudo, para Wuebbles e Hayhoe (2002) e Lima (2005), as áreas alagadas respondem por cerca de 30\% das emissões naturais biogênicas, algo entre 100 e $250 \mathrm{Tg} \mathrm{CH}_{4}$.ano ${ }^{-1}$, reforçando ainda mais o potencial emissor destas áreas. A Tabela 1 apresenta a contribuição das fontes naturais de metano para a atmosfera, com base nos dados do IPCC (2007).

Tabela 1. Fontes naturais e variabilidade global do metano atmosférico.

\begin{tabular}{l|c}
\hline \multicolumn{1}{c|}{ Fontes } & Tg $\mathbf{C H}_{\mathbf{4} \cdot \mathbf{a n o}^{\mathbf{- 1}}}$ \\
\hline Áreas Alagadas & $100-231$ \\
Cupins & $20-29$ \\
Oceanos & $4-15$ \\
Hidratos & $4-5$ \\
Fontes Geológicas & $4-14$ \\
Animais Selvagens & 15 \\
Queimadas & $2-5$ \\
Total & $\mathbf{1 4 5 - 2 6 0}$ \\
\hline
\end{tabular}

Fonte: Adaptado de IPCC (2007). 
Reconhecida como a lagoa mais urbanizada do município do Rio de Janeiro, a Lagoa Rodrigo de Freitas é abastecida por rios canalizados que conduzem a ela fluxos residuais e pluviais em quantidade e qualidade não controladas (FEEMA, 1995; Machado, 2009).

Esta observação se acentua nos dias de precipitação intensa, quando a lagoa recebe a descarga do sistema de águas pluviais e o esgoto doméstico que seria lançado ao mar por meio de emissário (Enrich-Prast et al., 2008).

Evidentemente, o eventual acúmulo de carga orgânica na Lagoa Rodrigo de Freitas pode fomentar alterações no fluxo de metano para a atmosfera, além de condições de desequilíbrio e degradação ambiental.

Nota-se, a reflexo disto, a ocorrência de períodos de mortandade de peixes, até então associados ao frágil equilíbrio promovido pelo acúmulo de matéria orgânica na Lagoa, às variações bruscas de sua salinidade (Oliveira et al., 1957) e à estratificação, que ocorre devido a insuficiente circulação e renovação de suas águas (Rosman et al., 1992).

Com relação à circulação das águas, salienta-se que a Lagoa Rodrigo de Freitas possui uma única ligação com o oceano através de um estreito canal com $835 \mathrm{~m}$ de extensão denominado Jardim de Alah, o qual permite a circulação da água entre estes ambientes, fazendo com que o balanço hídrico da Lagoa se dê por esta conexão (Lima, 2010). Todavia, a água do mar penetra na Lagoa somente no período da preamar das marés de sizígia e a troca de massas existente entre ela e o mar dá-se de maneira bastante restrita (Maciel, 2007).

Estudos geológicos realizados na década de 1970 identificaram uma falha disruptiva de grande porte na região da Lagoa Rodrigo de Freitas, denominada como Falha de São João, (Brenner et al.,1980; Rideg et al., 1989; Tupinambá et al., 2010), também indicada por anomalias do campo magnético na parte central da Lagoa, em levantamento realizado por Ponte Neto et al. (2010).

Embasado nestes estudos e em dados observacionais das falhas e lineamentos geológicos na área ao entorno da Lagoa, Hamza (2010) sugere que a ocorrência de depressões tectônicas poderia propiciar a liberação de gases e a descarga de fluídos termais de origem crustal na região, que, mesmo episódicos, dependendo da forma e intensidade a que ocorressem, determinariam a morte dos peixes por anoxia.

Enfim, faltam dados que permitam uma conclusão sobre a questão e os motivos que desencadeiam a morte dos peixes que ainda ocorre, uma vez que as intervenções antrópicas aumentam ano a ano e dificultam a aplicação de soluções sustentáveis para que melhorias na qualidade ambiental da área ocorram.

Neste trabalho apresentam-se os resultados de medidas de emissão de metano na Lagoa Rodrigo de Freitas, que objetivaram, de modo prospectivo, avaliar a relação entre algumas características físico-químicas básicas da água e o transporte de metano para a atmosfera na área da lagoa em dois dias típicos de verão na cidade do Rio de Janeiro.

\section{MATERIAIS E MÉTODOS}

A Lagoa Rodrigo de Freitas está localizada na cidade do Rio de Janeiro, entre as coordenadas $22^{\circ} 57^{\prime}$ e $22^{\circ} 58^{\prime}$ de latitude sul e $43^{\circ} 11^{\prime}$ e $43^{\circ} 13^{\prime}$ de longitude oeste (IBAMA, 1989). Seu formato atual é de um polígono irregular, com um espelho d'água que ocupa a área de $2,5 \mathrm{~km}^{2}$ e profundidade máxima próxima de $11 \mathrm{~m}$ (Andreata et al., 1997). O clima da região se enquadra no grupo "AW" da classificação de Köppen, apresentando verão úmido e inverno seco.

Utilizando-se de uma pequena embarcação que era ancorada nos pontos de coleta, foram realizadas duas campanhas em 2011: a primeira em 02 de fevereiro e a segunda em 15 de março, dias que se mostraram típicos de verão na cidade do Rio de Janeiro. Embora os registros de precipitação acumulada nos dias que antecederam as coletas tenham alcançado, 
BRAZ, L.; FERREIRA, W. J.; SILVA, M. G.; ALVALÁ, P. C.; MARANI, L.; BATISTA, G. T.; HAMZA, V. M. Influência de características físico-químicas da água no transporte de metano para a atmosfera na Lagoa Rodrigo de Freitas, RJ. Ambi-Agua, Taubaté, v. 7, n. 3, p. 99-112, 2012. (http://dx.doi.org/10.4136/ambiagua.972)

respectivamente, $50 \mathrm{~mm}$ e $22 \mathrm{~mm}$ (SINDA, 2012), no momento das observações ambos os dias estavam claros, com céu aberto e poucas nuvens, com a temperatura média de $29^{\circ} \mathrm{C}$, variando de 27 a $32^{\circ} \mathrm{C}$ durante os horários de coleta.

$\mathrm{Na}$ Figura 1 são ilustrados os cinco pontos amostrais deste trabalho, bem como é apresentada a batimetria da Lagoa, segundo dados do Projeto Ambiental Lagoa Limpa (Grupo EBX, 2011).

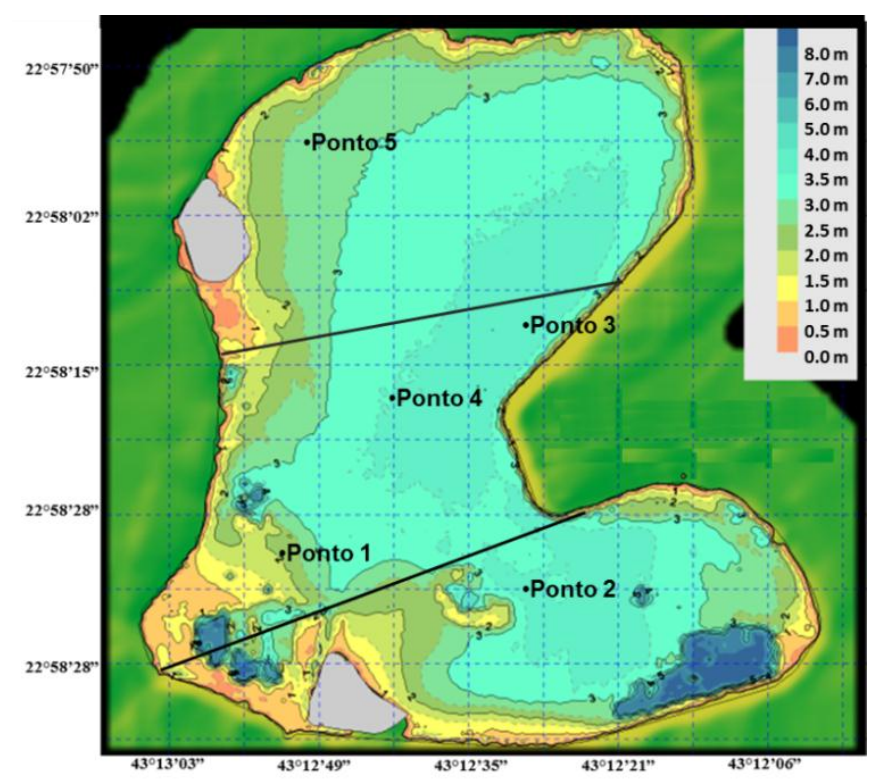

Figura 1. Batimetria da Lagoa Rodrigo de Freitas, RJ, e indicação dos pontos amostrais: as linhas contínuas delimitam a Falha de São João (Ponte Neto et al., 2010). Imagem adaptada do Projeto Ambiental Lagoa Limpa (Grupo EBX, 2011).

Os pontos de amostragem 1, 3 e 4 foram definidos a partir da falha geológica que cruza a Lagoa (Ponte Neto et al., 2010), enquanto os pontos 2 e 5 foram escolhidos por estarem fora desta faixa de delimitação. Registra-se que o ponto 1 foi situado junto à área de atividade de pescadores na Lagoa e o ponto 5 próximo a uma das entradas de dejetos e efluentes do sistema de esgoto.

As amostras foram obtidas por meio da técnica de câmara estática (Khalil et al., 1998; Marani e Alvalá, 2007), onde o metano emitido pela superfície da lagoa foi coletado em seringas plásticas de polipropileno com o uso de cúpulas cilíndricas de PVC, com volume de 26 litros e flutuadores de espuma acoplados em sua base para sustentação na água (Figura 2).

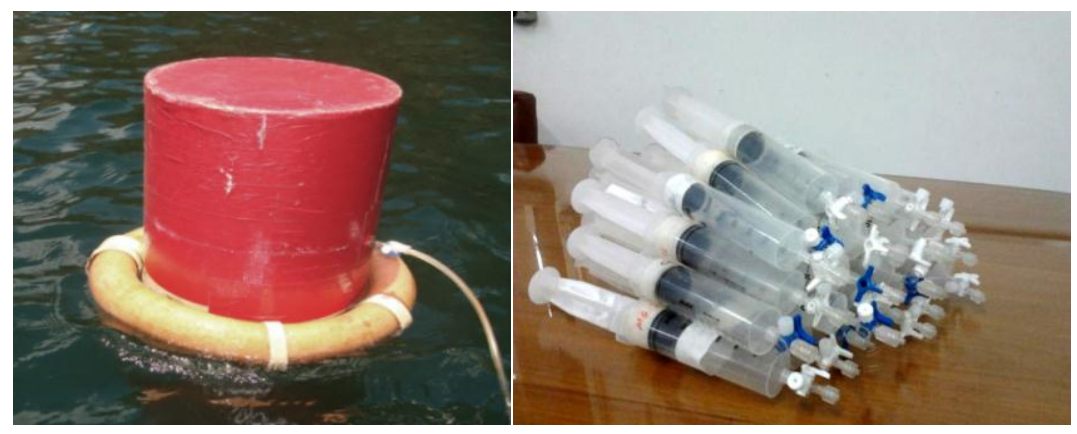

Figura 2. Câmara de PVC e seringas de polipropileno utilizadas nas coletas. 
Amostras de água foram coletadas nos pontos observando a batimetria da Lagoa (Figura 1 por meio de uma garrafa de Van Dorn. Utilizando-se a técnica de headspace (Ioffe e Vitenberg, 1984), a concentração de metano dissolvido foi determinada para estas amostras, sendo possível obter um perfil de metano dissolvido na coluna d'água da Lagoa com medidas em três profundidades: logo abaixo da superfície, no meio da coluna d'água e próximas ao sedimento no fundo da lagoa. Assim, com auxílio de seringas plásticas, foram retirados $30 \mathrm{ml}$ da água coletada em cada um dos níveis, sendo em cada uma delas adicionados $30 \mathrm{ml} \mathrm{de} \mathrm{ar}$ ambiente. Cada seringa foi agitada manualmente por aproximadamente dois minutos e permaneceu em repouso pelo mesmo período. Após isto, o ar de cada seringa foi injetado em um pequeno frasco de vidro previamente evacuado, com volume de $20 \mathrm{ml}$, e acondicionado para análise em laboratório. Destaca-se que o metano é pouco solúvel em água e, no momento da agitação, devido à variação da pressão parcial, o gás dissolvido na água passa para o ar existente na seringa.

As amostras foram enviadas ao Laboratório de Biogeoquímica Ambiental do INPE, São José dos Campos, SP, analisadas por meio da técnica de cromatografia gasosa associada a um detector de ionização de chama e as calibrações realizadas tendo por referência a concentração de um gás padrão confeccionado com $1804 \pm 2$ nanomol.mol ${ }^{-1}$ de $\mathrm{CH}_{4}$.

Nas análises foi injetado o volume total de cada seringa $(60 \mathrm{ml})$, sendo para cada uma delas executadas três injeções de $20 \mathrm{ml}$, considerada a média das três injeções como valor principal e o desvio padrão percentual entre as injeções de no máximo $1 \%$.

O fluxo de metano emitido pela lagoa foi obtido a partir da variação temporal da sua concentração no interior da câmara estática, descrita como a razão entre o número de moles de $\mathrm{CH}_{4}\left(\mathrm{n}_{\mathrm{CH}_{4}}\right)$ e o número total de moles da mistura $\left(\mathrm{n}_{\mathrm{t}}\right)$.

Adotando-se uma variação no número de moles de metano, $\Delta \mathrm{n}_{\mathrm{CH}_{4}}$, dado pela razão entre a massa da substância $\left(\mathrm{m}_{\mathrm{CH}_{4}}\right)$ e sua massa molar $\left(\mathrm{M}_{\mathrm{CH}_{4}}\right)$, ou seja, $\left(\mathrm{n}_{\mathrm{CH}_{4}}\right)=\mathrm{m}_{\mathrm{CH}_{4}} / \mathrm{M}_{\mathrm{CH}_{4}}$, e aplicando-se a equação dos gases ideais $(p V=m R T / M)$, pode-se escrever:

$$
\frac{\Delta \mathrm{n}_{\mathrm{CH}_{4}}}{\mathrm{n}_{\mathrm{t}}}=\frac{\Delta \mathrm{m}_{\mathrm{CH}_{4}} \mathrm{RT}}{\mathrm{M}_{\mathrm{CH}_{4}} \mathrm{pV}}=\Delta \chi_{\mathrm{CH}_{4}}
$$

Consequentemente,

$$
\Delta \mathrm{m}_{\mathrm{CH}_{4}}=\frac{\Delta \chi_{\mathrm{CH}_{4}} \mathrm{pVM}_{\mathrm{CH}_{4}}}{\mathrm{RT}}
$$

em que:

p é a pressão total (atm), $\mathrm{V}$ o volume total (litros), $\mathrm{M}_{\mathrm{CH}_{4}}$ é a massa molar do metano (16, $\left.04 \mathrm{~g} \mathrm{~mol}^{-1}\right)$, R é a constante dos gases $\left(0,082 \mathrm{~atm} \mathrm{~L} \mathrm{~mol}^{-1} \mathrm{~K}^{-1}\right)$ e $\mathrm{T}$ é a temperatura $(\mathrm{K})$. $\mathrm{O}$ termo $\Delta \chi_{\mathrm{CH}_{4}}$ faz referência à razão de mistura do metano.

Admitindo-se que a variação do fluxo por meio da área $\mathrm{A}\left(\mathrm{m}^{2}\right)$ seja constante durante um intervalo de tempo $\Delta \mathrm{t}$, descreve-se o fluxo $\Phi_{\mathrm{CH}_{4}}\left(\mathrm{mgCH}_{4} \mathrm{~m}^{-2} \mathrm{dia}^{-1}\right)$ como:

$$
\Phi_{\mathrm{CH}_{4}}=\frac{\Delta \chi_{\mathrm{CH}_{4}} \mathrm{pVM}_{\mathrm{CH}_{4}}}{\Delta \text { tART }}=\frac{p M_{\mathrm{CH}_{4}}}{\text { ART }} \frac{\Delta \chi_{\mathrm{CH}_{4}}}{\Delta \mathrm{t}}=\frac{\mathrm{pVM}_{\mathrm{CH}_{4}}}{\text { ART }} \frac{\partial \chi_{\mathrm{CH}_{4}}}{\partial \mathrm{t}}
$$


A variável mais importante na Equação 3 é $\partial \chi_{\mathrm{CH}_{4}} / \partial \mathrm{t}$, que é a derivada das medidas de concentração, estando todas as demais variáveis associadas às medições efetuadas diretamente no momento da coleta. Esta grandeza foi estimada a partir de uma regressão linear, considerando-se $\partial \chi_{\mathrm{CH}_{4}} / \partial \mathrm{t}$ como o "b" em $\chi=\chi_{0}+\mathrm{bt}$.

Enfim, para utilizar a regressão linear na obtenção de $\partial \chi_{\mathrm{CH}_{4}} / \partial \mathrm{t}$, considerou-se que o fluxo foi constante durante o tempo de coleta das amostras. Para afirmar a confiabilidade dos dados, devido à possibilidade de ocorrência de erro amostral e contaminação das amostras desde a etapa de coleta até a análise cromatográfica, foram adotados dois critérios de validação dos fluxos:

1. Observância do coeficiente de determinação $\left(\mathrm{r}^{2}\right)$ : foram considerados somente os valores de $\mathrm{r}^{2}$ superiores a 0,9 na reta de regressão para garantir a linearidade requerida dos pontos (Khalil et al., 1998; Alvalá e Kirchhoff; 2000).

2. Comparação entre a concentração inicial de metano no tempo inicial $(t=0)$, obtida a partir da regressão linear, e a concentração do metano em amostras de ar ambiente, coletadas antes de cada coleta: a concentração do metano nas amostras de ar ambiente e no tempo inicial da coleta devem apresentar valores semelhantes.

A fim de caracterizar os parâmetros físico-químicos na coluna d'água e verificar possíveis relações existentes entre estes parâmetros e o fluxo de metano, foram medidas as variáveis potencial hidrogeniônico $(\mathrm{pH})$, oxigênio dissolvido (OD), potencial de óxido redução (ORP) e temperatura do ar e da água com o uso de uma sonda de multiparâmetros de qualidade da água, marca Horiba, modelo U-51, que permitiu a tomada de medidas diretamente submerso na água e eliminou a necessidade de coletar amostras.

A análise dos componentes principais (ACP) foi usada para reduzir os dados físicoquímicos por combinações lineares das variáveis originais e julgar a importância destas próprias variáveis no processo de emissão de metano.

Uma vez que os parâmetros ambientais não variaram significativamente a $95 \%$ de confiabilidade pelo teste $\mathrm{t}$ de Student, na análise, os dados foram agrupados e avaliados por campanha, independentemente do ponto onde foram coletados.

Estes dados foram, então, transformados em um conjunto menor de combinações lineares, que responderam pela maior parte da variância do grupo inicial (Moita e Moita Neto, 1998; Cazar, 2003). Neste novo conjunto, reduziu-se o número de variáveis mantendo a informação original tanto quanto fosse possível, de forma que as variáveis com características semelhantes pudessem ser agrupadas em fatores. Uma matriz de correlação entre as o fluxo de metano e variáveis com maior peso na combinação linear das primeiras componentes principais foi gerada a partir destes fatores, determinando, assim, quais foram as variáveis medidas mais importantes do ponto visto estatístico.

\section{RESULTADOS E DISCUSSÃO}

Os resultados das campanhas realizadas nos dias 2 de fevereiro e em 15 de março de 2011, em que foram coletadas 200 amostras nos pontos interiores da Lagoa e que resultaram em 46 fluxos, dos quais 36 foram validados, indicam a variabilidade no fluxo do gás nos dois dias de amostragem. Os valores registrados nas campanhas 1 e 2 foram de $39 \pm 11$ e $29 \pm 6$ $\mathrm{mgCH}_{4} \mathrm{~m}^{-2} \mathrm{~d}^{-1} \mathrm{em}$ média, respectivamente, caracterizando, portanto, a Lagoa Rodrigues de Freitas como emissora de metano para a atmosfera.

Alterações no padrão de vento podem ter favorecido a dispersão dos valores do fluxo de metano, uma vez que, decorrente da ação dos ventos, a circulação da água tenderia a colocar em suspensão partículas sólidas e trazer parte dos poluentes já sedimentados de volta para a coluna d'água (Enrich-Prast et al., 2008). Contudo, ressalta-se que, em profundidades 
BRAZ, L.; FERREIRA, W. J.; SILVA, M. G.; ALVALÁ, P. C.; MARANI, L.; BATISTA, G. T.; HAMZA, V. M. Influência de características físico-químicas da água no transporte de metano para a atmosfera na Lagoa Rodrigo de Freitas, RJ. Ambi-Agua, Taubaté, v. 7, n. 3, p. 99-112, 2012. (http://dx.doi.org/10.4136/ambiagua.972)

menores do que dois metros, a mistura das massas de água ocorre somente nos casos de vento moderado, predominantemente observados nos dias de coleta.

A emissão média geral da lagoa foi de $33 \pm 10 \mathrm{mgCH}_{4} \mathrm{~m}^{-2} \mathrm{~d}^{-1}$, fluxo médio que é menor, porém condizente com os resultados apresentados por estudos realizados em lagoas em condição semelhante de uso: lagoas urbanas construídas para tratamento de águas residuais em zonas úmidas da Nova Zelândia apresentam valores que variam de 45 a $526 \mathrm{mgCH}_{4} \mathrm{~m}^{-2} \mathrm{~d}^{-1}$ (Tanner et al., 1997), enquanto na Suécia, estima-se que este valor esteja entre 80 e 180 $\mathrm{mgCH}_{4} \mathrm{~m}^{-2} \mathrm{~d}^{-1}$ (Johansson et al., 2004).

$\mathrm{Na}$ Tabela 2 são apresentados os fluxos de metano obtidos por meio das câmaras estáticas na interface entre a água e a atmosfera nestas duas campanhas.

Tabela 2. Fluxo de metano na interface água-atmosfera na Lagoa Rodrigo de Freitas, RJ.

\begin{tabular}{c|c|c|c|c|c|c}
\hline \multirow{2}{*}{ Campanha } & \multirow{2}{*}{ Ponto } & \multirow{2}{*}{ Prof. (m) } & \multicolumn{4}{|c}{ Fluxo de metano $\left(\mathbf{m g C H}_{\mathbf{4}} \mathbf{m}^{-\mathbf{2}} \mathbf{d}^{-\mathbf{1}}\right)$} \\
\cline { 3 - 6 } & & $\mathbf{N}$ & Média & $\mathbf{D P}$ & $\mathbf{D P \%}$ \\
\hline 1 & 1 & 2,0 & 5 & 52,7 & 5,8 & 11,0 \\
1 & 2 & 4,5 & 4 & 27,4 & 2,4 & 8,7 \\
1 & 3 & 3,5 & 5 & 28,7 & 2,7 & 9,5 \\
1 & 4 & 3,5 & 4 & 36,4 & 3,2 & 8,7 \\
1 & 5 & 2,5 & 4 & 47,6 & 2,0 & 4,2 \\
2 & 1 & 2,0 & 3 & 36,6 & 0,6 & 1,6 \\
2 & 2 & 4,5 & 2 & 22,0 & 1,5 & 6,8 \\
2 & 3 & 3,5 & 3 & 29,7 & 1,9 & 6,4 \\
2 & 4 & 3,5 & 3 & 23,5 & 2,2 & 9,2 \\
2 & 5 & 2,5 & 3 & 31,1 & 2,9 & 9,4 \\
\hline
\end{tabular}

*N: número de fluxos válidos; DP: desvio padrão; DP\%: desvio padrão percentual.

Coletado na zona da falha, no Ponto 1 foi registrado o maior fluxo médio de metano entre as campanhas, $44,7 \pm 11,4 \mathrm{mgCH}_{4} \mathrm{~m}^{-2} \mathrm{~d}^{-1}$, ao passo que no Ponto 2, observou-se o menor fluxo médio do experimento, 24,7 $\pm 3,8 \mathrm{mgCH}_{4} \mathrm{~m}^{-2} \mathrm{~d}^{-1}$. Nos outros pontos, independentemente de sua localização dentro ou fora da falha, não houve uma variação significativa na medida do fluxo de metano, tornando impossível caracterizar, neste trabalho, a possível influência da Falha de São João na emanação do metano.

Há de se considerar que os maiores valores de emissão, observados em ambas as campanhas nos Pontos 1 e 5, podem estar relacionados às fontes antrópicas locais, como a atividade pesqueira e a entrada de dejetos urbanos na área lagunar.

É pertinente reforçar que a precipitação observada nos dias de verão ocasionaria um maior aporte de esgoto à Lagoa, mas, no entanto, nos momentos de coleta não se observou esta ocorrência.

Embora fosse importante analisar a influência da variável precipitação no processo de emissão de metano, não foi escopo deste trabalho a avaliação deste aporte, uma vez que este item surgiria como um elemento bastante complexo para uma pesquisa transversal e exploratória.

\subsection{Medidas ambientais}

A Tabela 3 apresenta os valores amostrados de parâmetros físico-químicos medidos na água da Lagoa Rodrigo de Freitas. 
BRAZ, L.; FERREIRA, W. J.; SILVA, M. G.; ALVALÁ, P. C.; MARANI, L.; BATISTA, G. T.; HAMZA, V. M. Influência de características físico-químicas da água no transporte de metano para a atmosfera na Lagoa Rodrigo de Freitas, RJ. Ambi-Agua, Taubaté, v. 7, n. 3, p. 99-112, 2012. (http://dx.doi.org/10.4136/ambiagua.972)

Tabela 3. Parâmetros físico-químicos superficiais medidos na lagoa.

\begin{tabular}{|c|c|c|c|c|c|c|c|}
\hline \multirow{2}{*}{ Campanha } & \multirow{2}{*}{ Ponto } & Profundidade & $\mathbf{T}$ ar & $\mathrm{T}$ água & pH & ORP & OD \\
\hline & & $\mathbf{m}$ & \multicolumn{3}{|c|}{${ }^{\circ} \mathbf{C}$} & $\mathbf{m V}$ & $\mathrm{mg} / \mathrm{L}$ \\
\hline 1 & 1 & 2 & 29,8 & 31,0 & 7,0 & 105,0 & 2,9 \\
\hline 1 & 2 & 4,5 & 29,4 & 30,8 & 8,0 & 58,0 & 5,4 \\
\hline 1 & 3 & 3,5 & 30,6 & 32,0 & 7,8 & 65,0 & 5,3 \\
\hline 1 & 4 & 3,5 & 31,6 & 32,0 & 8,0 & 66,0 & 5,8 \\
\hline 1 & 5 & 2,5 & 31,9 & 33,1 & 8,3 & 52,0 & 6,3 \\
\hline 2 & 1 & 2 & 27,5 & 29,5 & 7,8 & 65,0 & 4,3 \\
\hline 2 & 2 & 4,5 & 27,6 & 28,8 & 8,1 & 56,9 & 6,2 \\
\hline 2 & 3 & 3,5 & 27,4 & 28,8 & 7,7 & 80,0 & 4,3 \\
\hline 2 & 4 & 3,5 & 27,7 & 28,8 & 7,5 & 88,0 & 3,4 \\
\hline 2 & 5 & 2,5 & 29,8 & 29,1 & 7,7 & 63,0 & 4,1 \\
\hline
\end{tabular}

*T ar: temperatura do ar; T água: temperatura da água; ORP: potencial de óxido redução; OD: oxigênio dissolvido.

Corroborando Marani e Alvalá (2007), observou-se um indicativo de relação existente entre a profundidade, dada como a altura medida do espelho d'água até o fundo da lagoa, e a emissão de metano.

De modo geral, foram observadas menores emissões em áreas mais profundas da Lagoa, fato que foi associado à queda na taxa de difusão vertical do metano decorrente do aumento da pressão com a profundidade na coluna de água e à presença de bactérias metanotróficas, que consomem o metano ao longo da coluna de água (Pellizari et al., 2007).

A partir dos dados da Tabela 3 observou-se que os parâmetros medidos na Lagoa se encontravam dentro da faixa de eficiência de produção de metano em meio aquoso, apresentados, como referência, na Tabela 4.

Tabela 4. Eficiência de produção de $\mathrm{CH}_{4}$ em relação aos parâmetros ambientais.

\begin{tabular}{l|c|l}
\hline Parâmetros & Faixa de variação & \multicolumn{1}{c}{ Referência } \\
\hline T Água & 15 a $37^{\circ} \mathrm{C}$ & Yang e Chang (1998) \\
$\mathrm{pH}$ & 6,4 a 7,8 & Wang et al. (1993) \\
OD & $<10 \mathrm{mg} / \mathrm{L}$ & Silva (2010) \\
ORP & -150 a $440 \mathrm{mV}$ & Wang et al., (1993); Silva (2010) \\
\hline
\end{tabular}

As temperaturas do ar e da água não apresentaram variação significativa, mantendo-se nos dois dias de coleta próxima a $28^{\circ} \mathrm{C}$ e $29^{\circ} \mathrm{C}$, respectivamente. A média do $\mathrm{pH}$ obtida na Lagoa Rodrigo de Freitas foi de 7,8, valor situado no limiar da faixa ótima de produção. Salienta-se que grande parte dos organismos produtores de metano é sensível ao $\mathrm{pH}$ e habitam locais onde, preferencialmente, sua condição é próxima da neutralidade (Wang et al., 1993).

Na primeira campanha, no Ponto 1, ponto de maior emissão de metano, observou-se a menor concentração de oxigênio dissolvido e a neutralidade do $\mathrm{pH}$. Neste ponto, o ORP, parâmetro utilizado como indicador da quantidade de matéria orgânica disponível no meio, mostrou-se mais elevado em relação aos outros pontos. Langmuir (1997) avalia que quanto 
maior o valor do ORP, mais favorecido se torna a produção de metano por arquéias metanogênicas. Sua diminuição, ao contrário, aliada ao aumento gradativo da concentração de oxigênio ao longo da coluna d'água proporcionaria incremento no consumo do metano antes que o mesmo atingisse a superfície.

Silva (2010) afirma que a entrada de matéria orgânica na planície sul pantaneira devido ao regime hídrico local faz com que o $\mathrm{pH}$ decaia de 8 no período de seca para 3 na época de cheia, denotando-a como um dos maiores agentes redutores em áreas alagadas.

Diante disto, quando verificada uma maior quantidade de matéria orgânica no sistema, há uma tendência de o pH projetar-se mais ácido e se observar a elevação do consumo de oxigênio, o que, consequentemente, torna o ORP mais positivo.

Nos dados da Lagoa Rodrigo de Freitas ambas as prospecções foram observadas e, de modo geral, os parâmetros pH, ORP e OD sinalizam uma baixa quantidade de matéria orgânica disponível na Lagoa, uma vez que não houve alteração significativa destes parâmetros na maior parte do experimento. Esta informação é reforçada pelo estudo de Baptista Neto et al. (2011), que verificou que os sedimentos de fundo da Lagoa Rodrigo de Freitas são predominantemente lamosos, estando a concentração de matéria orgânica entre 0 e $35 \%$ do peso total da amostra. Segundo estes autores, percentuais mais baixos são registrados no entorno do Jardim de Alah, que sofre uma maior influência hidrodinâmica e denota a presença de sedimentos arenosos no local.

Outrossim, a análise de componentes principais demonstrou que não seriam as variáveis $\mathrm{pH}$, ORP e OD os fatores mais importantes nos fluxos de metano observados.

\subsection{Análise das componentes principais}

Para a análise dos componentes principais (ACP) tomou-se inicialmente um conjunto de dados com cinco variáveis e 36 amostras, de onde puderam ser retidas as componentes principais com autovalores menores do que 1 .

A CP1 indicou que $57 \%$ da variância dos dados são explicados por três componentes principais (pH, ORP e OD), enquanto a $\mathrm{CP} 2$, representando $87 \%$ da variância das amostras, foi explicada por duas componentes (profundidade e temperatura). A partir da Tabela 5, nos Grupos CP1 e CP2, são apresentados os pesos estatísticos que foram utilizados na diminuição do número de variáveis da análise de componentes principais.

Tabela 5. Análise de Componentes Principais dos parâmetros subsuperficiais medidos na coluna d'água da Lagoa Rodrigo de Freitas.

\begin{tabular}{l|c|c}
\hline Parâmetros & CP1 & CP2 \\
\hline Profundidade & $-0,52$ & $-0,71$ \\
Temperatura & $-0,41$ & 0,81 \\
pH & $-0,97$ & 0,00 \\
ORP & $-0,92$ & 0,01 \\
OD & $-0,98$ & 0,04 \\
\hline
\end{tabular}

Após determinar novos fatores de acordo com os grupos CP1 e CP2, foi gerada uma matriz de correlação entre estes fatores e o fluxo médio de metano obtido por ponto medido. Notou-se que o grupo que melhor poderia explicar a variação do fluxo de metano na Lagoa Rodrigo de Freitas foi o CP2, o qual se fundamenta nas medidas de profundidade e temperatura. A partir destes dados obteve-se a curva de regressão linear entre o fluxo médio de metano e $\mathrm{CP} 2$, cujo $\mathrm{R}^{2}$ mostrou-se significativo, com valor de 0,87 , revelando a influência 
BRAZ, L.; FERREIRA, W. J.; SILVA, M. G.; ALVALÁ, P. C.; MARANI, L.; BATISTA, G. T.; HAMZA, V. M. Influência de características físico-químicas da água no transporte de metano para a atmosfera na Lagoa Rodrigo de Freitas, RJ. Ambi-Agua, Taubaté, v. 7, n. 3, p. 99-112, 2012. (http://dx.doi.org/10.4136/ambiagua.972)

destes parâmetros na emissão de metano pela Lagoa. A Figura 3 ilustra este resultado, bem como o limite de confiabilidade da curva a $95 \%$ de confiança.

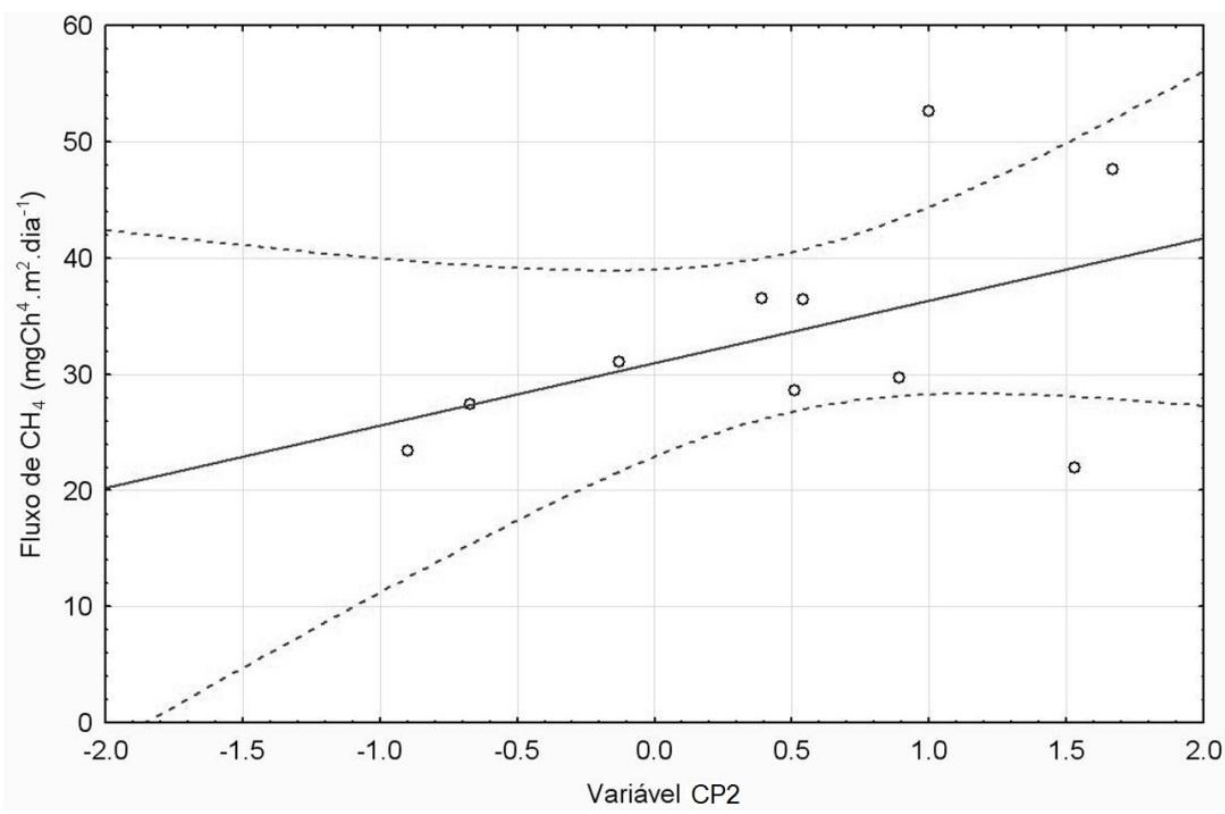

Figura 3. Regressão linear e limite de confiabilidade a $95 \%$ entre o fluxo médio de metano e as variáveis do Grupo $\mathrm{CP} 2$, profundidade e temperatura.

Vale ressaltar que na relação observada, o reduzido número de amostras válidas obtidos em dois dias de avaliação tornou limitada qualquer inferência estatística que pudesse aprofundar a análise destes resultados, sendo necessária uma maior quantidade de fluxos, com novas observações em períodos sazonais diferenciados, para que se seja determinado o quanto estas variáveis poderão influenciar nas emissões de metano.

Assim, ganha importância a organização de um maior número de campanhas para obtenção de dados para o estudo da variação sazonal das emissões, bem como informações sobre a variação climática da região, principalmente, porque ocorrem mudanças nos fluxos residuais e pluviais na área da Lagoa.

\subsection{Metano dissolvido e variáveis ambientais}

Para o estudo do metano dissolvido foram coletadas 30 amostras na coluna d'água da Lagoa Rodrigo de Freitas. Observou-se que o perfil médio do metano dissolvido na Lagoa apresentou valores crescentes partindo da superfície até o fundo (Tabela 6).

Tabela 6. Variabilidade do metano dissolvido na coluna d'água da Lagoa Rodrigo de Freitas.

\begin{tabular}{cc|c}
\hline \multicolumn{2}{c|}{$\begin{array}{c}\text { Profundidade } \\
(\mathbf{m})\end{array}$} & $\begin{array}{c}\text { Metano Dissolvido } \\
\left(\mathbf{m g L}^{-1}\right)\end{array}$ \\
\hline Sub-superfície & $(0,0-0,5)$ & $0,010 \pm 0,002$ \\
Meio & $(0,5-2,0)$ & $0,013 \pm 0,003$ \\
Fundo & $(2,0-4,5)$ & $0,020 \pm 0,009$ \\
\hline
\end{tabular}


BRAZ, L.; FERREIRA, W. J.; SILVA, M. G.; ALVALÁ, P. C.; MARANI, L.; BATISTA, G. T.; HAMZA, V. M. Influência de características físico-químicas da água no transporte de metano para a atmosfera na Lagoa Rodrigo de Freitas, RJ. Ambi-Agua, Taubaté, v. 7, n. 3, p. 99-112, 2012. (http://dx.doi.org/10.4136/ambiagua.972)

$\mathrm{Na}$ Tabela 7 são ilustrados os parâmetros físico-químicos médios na coluna d'água dos cinco pontos amostrais medidos neste ensaio, obtidos na subsuperfície, no meio e no fundo da Lagoa.

Tabela 7. Perfil dos parâmetros ambientais medidos na coluna d'água da Lagoa ( $\mathrm{n}=5$ pontos)

\begin{tabular}{l|c|c|c|c|c}
\hline \multicolumn{1}{c|}{ Posição } & Parâmetros & T água (-C) & $\mathbf{p H}$ & ORP $(\mathbf{m V})$ & OD $\left(\mathbf{m g ~ L}^{-\mathbf{1}} \mathbf{)}\right.$ \\
\hline \multirow{4}{*}{ Sub-superfície } & Média & 29,0 & 7,8 & 70,6 & 4,5 \\
& Dp & 0,3 & 0,2 & 12,9 & 1,0 \\
& Dp\% & 1 & 3 & 18 & 23 \\
\hline \multirow{3}{*}{ Meio } & Média & 28,8 & 7,7 & 73,4 & 3,9 \\
& Dp & 0,5 & 0,2 & 13,0 & 1,0 \\
& Dp\% & 2 & 3 & 18 & 26 \\
\hline \multirow{3}{*}{ Fundo } & Média & 28,6 & 7,6 & 78,4 & 3,2 \\
& Dp & 0,2 & 0,3 & 15,4 & 1,3 \\
& Dp\% & 1 & 4 & 20 & 40 \\
\hline
\end{tabular}

A partir da Tabela 7 verificou-se que a temperatura da água e o $\mathrm{pH}$ se mantiveram constantes em relação à batimetria da lagoa. No entanto, o OD aumentou na medida em que se aproximava da superfície, sendo incrementado pelas trocas gasosas que ocorriam no limiar da interação da água com a atmosfera.

Diferentemente do OD, o ORP apresentou valor mais elevado quando medido próximo ao sedimento, fato que pode ser justificado pela maior concentração de matéria orgânica no fundo da lagoa, o que tende a consumir o oxigênio dissolvido tornando o ambiente mais redutor na interface entre a água e o sedimento.

Ainda por meio da Tabela 7 foi reforçada a ideia de que em ambientes menos oxigenados ocorrem indícios de favorecimento à produção do metano, pois, onde a concentração de oxigênio dissolvido foi menor, consequentemente coexistindo um potencial redutor mais elevado, somente uma fração do gás produzido na interface entre a água e o sedimento foi oxidada por micro-organismos, sendo o saldo residual transferido para atmosfera ao longo da coluna de água.

A partir do estudo do metano dissolvido na Lagoa Rodrigo de Freitas verificou-se que nem todo o metano produzido próximo ao substrato chega a ser transportado para a superfície, justificando os reduzidos valores de fluxo registrados neste trabalho.

\section{CONCLUSÃO}

As medidas de fluxo de metano realizadas na Lagoa Rodrigo de Freitas, resultantes de um estudo prospectivo com referência em dois dias típicos de verão, revelaram-na como uma fonte emissora desse gás para a atmosfera, com fluxo médio de $33 \pm 10 \mathrm{mgCH}_{4} \mathrm{~m}^{-2} \mathrm{~d}^{-1}$. Esse valor é próximo do observado em lagoas de semelhante utilização.

Independentemente da localização dos pontos amostrais, dentro ou fora da falha, não houve uma variação significativa na medida do fluxo de metano, tornando impossível caracterizar, neste trabalho exploratório, a possível influência da Falha de São João na emanação do metano.

A profundidade, medida associada à altura da lâmina d'água até o fundo da lagoa, junto à temperatura revelaram-se como os parâmetros de maior influência no processo de emissão atmosférica do metano pela Lagoa.

A análise do perfil de metano na coluna d'água sugere uma maior concentração do gás nos estratos sedimentares do fundo da Lagoa, apontado por medidas de $\mathrm{pH}$, oxigênio 
dissolvido e ORP ao longo da coluna. Porém, nem todo o metano produzido chega a ser transportado à superfície, sendo consumido na medida em que se aproxima dela.

Deve-se ressaltar a existência de uma complexidade na limnologia da água da Lagoa em razão dos diferentes agentes que podem interferir nas emissões atmosféricas.

\section{AGRADECIMENTOS}

Ao apoio financeiro da Fundação de Amparo à Pesquisa do Estado do Rio de Janeiro FAPERJ, por meio do Projeto "Caracterização Físico-Química das Emanações provenientes das Falhas Geológicas na área Costeira do Estado do Rio de Janeiro" (Processo $n^{\circ}$. 111.342/2010).

\section{REFERÊNCIAS}

ALVALÁ, P. C.; KIRCHHOFF, W. V. J. H. Methane fluxes from the Pantanal floodplain in Brazil: seasonal variation. In: van Ham et al. (Eds.) Non- $\mathrm{CO}_{2}$ Greenhouse gases: scientific understanding, control and implementation. Kluwer Academic Publishers, 2000, p. 95-99.

ANDREATA, J. V.; MARCA, A. G.; SOARES C. L.; SANTOS R. S. . Distribuição mensal dos peixes mais representativos da Lagoa Rodrigo de Freitas. Rio de Janeiro, Brasil. Revista Brasileira de Zoologia, 1997, 14 (1): pp. 121-13.

BAPTISTA NETO, J. A.; SILVA, C. G.; DIAS, G. T. M.; FONSECA, E. M. Distribuição sedimentar da Lagoa Rodrigo de Freitas através de sísmica de alta resolução. Revista Brasileira de Geofísica. [online]. 2011, Vol. 29, n. 1, pp. 187-195. ISSN 0102-261X. http://dx. doi. org/10. 1590/S0102-261X2011000100013.

BRENNER, X; FERRARI, Y; PENHA, Z. Anais do XXXI Congresso Brasileiro de Geologia, 1980, v. 5.

CAZAR, R. A. An exercise on chemometrics for a quantitative analysis course. Journal of. Chemical Education2003, Ed. 80(9): 1026-1029.

DALAL, R. C.; ALLEN, D. E. Greenhouse gas fluxes from natural ecosystems. Australian Journal of Botany, 2008, v. 56, p. 369-407.

ENRICH-PRAST, A.; BENTO, L.; SANTORO, A. Influência das Mudanças Globais sobre as Lagoas da Cidade do Rio de Janeiro. In: Sergio Besserman Vianna; Paulo Pereira Gusmão; Paula Serrano. (Org.). RIO Próximos 100 anos: O aquecimento global e a Cidade. RIO Próximos 100 anos: O aquecimento global e a Cidade. Rio de Janeiro: Instituto Pereira Passos, 2008, p. 176-185.

FEEMA. Lagoa Rodrigo de Freitas - Ações propostas para melhoria da Bacia. Relatório final do grupo de trabalho criado pelo decreto $\mathrm{n}^{\mathbf{0}} 13539.1995$.

GRUPO EBX. Projeto ambiental Lagoa Limpa: uma nova lagoa para o Rio de Janeiro. Disponível em: <http://www.lagoalimpa.com.br/>. Acesso em: 15 nov. 2011.

HAMZA, V. M. Episodes of Biotic Crisis in Coastal Lagoons of Rio de Janeiro: Similarities with Mass Extinctions of end-Permian and end-Cretaceous times, (Extended Abstract), Meeting of the Americas. Anais. 2010, Foz de Iguaçu, Brazil. 
IBAMA. Lagoa Rodrigo de Freitas. Segundo Ministério do interior. Instituto Brasileiro do Meio Ambiente e dos Recursos Naturais Renováveis. IBAMA - RJ, 1989, 177p.

IOFFE, B. V.; VITENBERG, A. G. Headspace Analysis and Related Methods in Gas Chromatography. John Wiley and Sons. 1984, 304p.

IPCC. INTERGOVERNMENTAL PANEL ON CLIMATE CHANGE. Climate Change 2007: The Physical Basis. Contribution of working Group I to the Fourth Assessment Report of the Intergovernmental Panel on Climate Change. Cambridge. 2007.

JOHANSSON, A. E.; GUSTAVSSON, A. M.; ÖQUIST, M. G.; SVENSSON, B. H. Methane emissions from a constructed wetland treating wastewater: Seasonal and spatial distribution and dependence on edaphic factors. Water Research2004, 38:3960-3970.

KHALIL, M. A. K.; RASMUSSEN, R. A.; SHEARER, M.; DALlUGE, R.; REN, L.; DUAN, C. L. Factors affecting methane emissions from rice fields. Journal of Geophysical Research, 1998, v. 103, n. D19, p. 25219-25231.

LANGMUIR, D. Aqueous environmental geochemistry. Upper Saddle River, NJ: PrenticeHall, Inc. 1997, 600p.

LIMA, I. B. T. Biogeochemical distinction of methane releases from two Amazon hydroreservoirs. Chemosphere, 2005, v. 59, p. 1697-1702.

LIMA, L. S. Estudos de hidrodinâmica ambiental e mudanças na qualidade das águas da Lagoa Rodrigo de Freitas após ligação com o mar via dutos afogados. 2010, 116p. Dissertação de Mestrado do Programa de Engenharia Oceânica da COPPE/UFRJ, Rio de Janeiro - RJ, 2010.

MACHADO, L. B. Evolução da degradação ambiental da bacia hidrográfica da Lagoa Rodrigo de Freitas - RJ. 2009, 178p. Dissertação (Mestrado em Engenharia Ambiental) - Universidade do Estado do Rio de Janeiro. Rio de Janeiro, 2009.

MACIEL, G. L. R. Análise de Dados Hidrodinâmicos e Estratificação na Lagoa Rodrigo de Freitas, RJ: Relação Com a Concentração de Oxigênio Dissolvido. 2007. Dissertação de M. Sc., COPPE / UFRJ, Rio de Janeiro, 2007.

MARANI, L.; ALVALÁ, P. C. Methane emissions from lakes and floodplains in Pantanal, Brazil. Atmospheric Environment, 2007, v. 41, p. 1627-1633.

MOITA, G. C.; MOITA NETO, J. M. Uma Introdução à análise exploratória de dados multivariados. Química Nova, 1998, 21(4): 467-469.

OLIVEIRA, L.; NASCIMENTO, R.; KRAU, L; MIRANDA， N. Observações hidrobiológicas e mortandade de peixes na Lagoa Rodrigo de Freitas. Memórias do Instituto Oswaldo Cruz, Rio de Janeiro, 1957, 55 (2):211-275.

PELLIZARI, V. H.; NAKAYAMA, C. R.; ARAUJO, A. C. V.; LIOTTI R. G.; VAZOLLER, R. F. Metano, Mudanças Climáticas Globais e a Microbiologia. Microbiologia In Foco, São Paulo. 2007, p. 4-9.

PONTE NETO, C. F.; VIEIRA, F. P.; HAMZA, V. M. Resultados de levantamentos geomagnéticos da região da Lagoa Rodrigo de Freitas. Relatório Interno Observatório Nacional. 2010. 
RIDEG, P.; STEIN, J.; TOTIS, E. A Falha do Morro de São João em Botafogo - Rio de Janeiro. Simpósio Regional de Geologia, 1, 1989, Rio de Janeiro. Sociedade Brasileira Geologia. Boletim de Resumos: 130-131.

ROSMAN, P. C. C.; NEVES, C. F.; MELO, E. Solução Conjunta dos Problemas de Erosão na Praia de Ipanema-Leblon e Qualidade de Água na Lagoa Rodrigo de Freitas. In: Relatório Final. COPPETEC, Rio de Janeiro-RJ, Brasil. 1992.

SILVA, M. G. Variação interanual e análise da influência de parâmetros ambientais na emissão de metano no Pantanal. 2010, 99p. Dissertação (Mestrado em Geofísica Espacial) - Instituto Nacional de Pesquisas Espaciais. São José dos Campos, 2010.

SINDA. Sistema Nacional de Dados Ambientais. INPE - Centro Regional Nordeste, Natal, RN. Dados disponíveis em <http://sinda.crn2.inpe.br>. Acesso em outubro de 2012.

TANNER, C. C.; ADAMS, D. D.; DOWNES, M. T. Methane emissions from constructed wetlands treating agriculturalwastewaters. Journal of Environmental Quality. 1997, v. 26, 1056-1062.

TUPINAMBÁ, M.; VIEIRA, F. P.; HAMZA, V. M. Resultados de levantamentos geológicos da região da Lagoa Rodrigo de Freitas. Relatório Interno, Observatório Nacional. 2010 .

WANG, Z. P.; DELAUNE, R. D.; MASSCHELEYN, P. H. (1993). Soil redox and pH effects on methane production in a flooded rice. Soil Science Society of America Journal, 1993 , v. 57, n. 2, pp. 382-38.

WUEBBLES, D. J.; HAYHOE, K. Atmospheric methane and global change. Earth-Science Reviews, 2002, v. 57, p. 177-210.

YANG, S. S.; CHANG, H. L. Effect of environmental conditions on methane production and emission from paddy soil. Agriculture Ecosystems \& Environment, Amsterdam, 1998, v. 69, n. 1, p. 69-80. 